\title{
Diversity - diskutieren oder tabuisieren? Eine explorative Studie in Indien
}
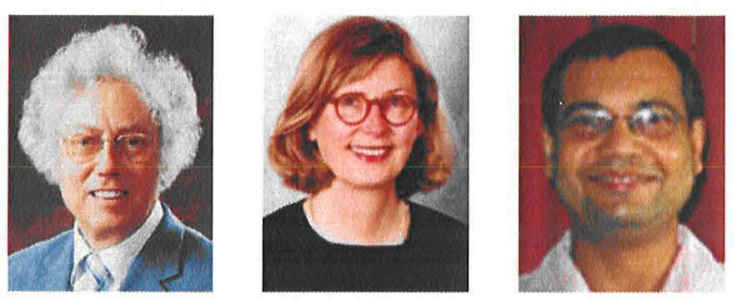

Diether Gebert • Sabine Boerner • Debabrata Chatterjee

Zusammenfassung: In Ergänzung zu vorliegenden Unterscheidungen innerhalb der DiversityForschung wird hier erstmals die Unterscheidung einer tabuisierten von einer nicht-tabuisierten Diversity eingeführt. Die Legitimierung dieser Unterscheidung ergibt sich daraus, dass - so die zentrale Annahme - tabuisierte Diversity mit einer Konfliktverschiebung, nicht-tabuisierte Diversity dagegen mit einer Konflikteskalation einhergeht. Für die Praxis des Managements ist diese Unterscheidung wichtig, um die Risiken des globalen Trends zu mehr Diversity genauer einschätzen zu können. An einer Stichprobe von N=96 Schulen im Bundesstaat Kerala in Südindien zeigte sich im Sinne der Konflikteskalations-These, dass die nicht-tabuisierte gewerkschaftliche Diversity mit gruppeninternen Spannungen positiv beschleunigt verbunden ist. Im Sinne der Konfliktverschiebungs-These zeigte sich, dass die nicht-tabuisierte gewerkschaftliche Diversity mit gruppeninternen Konflikten besonders eng verbunden ist, wenn zugleich die tabuisierte religiöse Diversity hoch ausgeprägt ist. Zur Erklärung der Befunde wird einerseits auf die Besonderheiten der Stichprobe in Kerala eingegangen. Zur Formulierung von Managementimplikationen wird andererseits präzisiert, in Bezug auf welche Diversity-Arten in Deutschland analoge Konstellationen vorzuliegen scheinen und welche Konsequenzen hieraus für das Management erwachsen.

\section{Prof. Dr. D. Gebert ( $\triangle)$}

Renmin University of China, School of Business,

100872 P. R. China

E-Mail: Diether.Gebert (4tu-berlin.de

Prof. Dr. S. Boerner

Fachbereich Politik- und Verwaltungswissenschaft, Lehrstuhl für Management, insbes.

Strategie und Führung, Universität Konstanz, Konstanz, Deutschland

E-Mail: Sabine.boerner@uni-konstanz.de

Dr. D. Chatterjee

Indian Institute of Management Kozhikode (IIMK), Kozhikode, India

E-Mail: Deb.chattopadhyay (a) gmail.com 
Schlüsselwörter: Religiöse Diversity · Gewerkschaftliche Diversity · Tabuisierung · Diversity Management

JEL Classification: M16 M50 $\cdot$ M54

\section{Problemstellung und Ziele der Untersuchung}

Im Zuge steigender Globalisierung und Internationalisierung nimmt die Heterogenität von Organisationsmitgliedern in Bezug auf Nationalität, Ausbildung, fachliche Ausrichtung, Betriebszugehörigkeit u. ä. tendenziell zu. ${ }^{1}$ In der Erwartung erhöhter Leistungsfähigkeit (wie z. B. Innovativität), aber auch unter der Perspektive eines modernen FairnessErwartungen gerecht werdenden Diversity-Managements ${ }^{2}$, wird die Heterogenität von organisationalen Teams zusätzlich aktiv gefördert ${ }^{3}$.

Ergebnisse der internationalen Forschung zeigen indes, dass sich Diversity in Organisationen als Vorteil, aber auch als Nachteil erweisen kann ${ }^{4}$. Einerseits kann Diversity durch die Erhöhung der Perspektiven-Vielfalt in der Gruppe Prozesse der Informationsverarbeitung und Entscheidungsfindung positiv beeinflussen. ${ }^{5}$ Andererseits kann Diversity jedoch über Prozesse der sozialen Kategorisierung Konflikte auslösen, die die Leistung der Gruppe beeinträchtigen. ${ }^{6}$ In der Tradition dieser letzteren Forschungslinie fokussieren wir in dieser Studie als abhängige Variable gruppeninterne Konflikte, die sich - so unsere Definition - in Form von Subgruppenbildungen und emotionalen Spannungen zwischen diesen Subgruppen dokumentieren. Unser Untersuchungsfokus liegt also nicht auf der Analyse von Chancen, sondern auf der Analyse von Risiken von Diversity, mit denen das Management konfrontiert wird.

In der Literatur werden sichtbare Formen von Diversity (z. B. Unterschiede im Alter) von weniger sichtbaren Formen von Diversity (z. B. Unterschiede bezüglich grundlegender Überzeugungen) voneinander unterschieden ${ }^{7}$; darüber hinaus wird eine stärker die kognitive Ebene betreffende aufgabenbezogene Diversity (z. B. Unterschiede im Bildungshintergrund) von einer stärker die emotionalen, zwischenmenschlichen Beziehungen berührenden Diversity (z. B. Unterschiede im Geschlecht) unterschieden. Bezüglich der Induktion leistungsbehindernder Konflikte erweisen sich Formen der sichtbaren und beziehungsbezogenen Diversity tendenziell als konfliktträchtiger als andere Diversity-Formen. ${ }^{\mathrm{s}}$

Im Sinne einer Ausdifferenzierung dieser Typologie von Diversity-Formen fügen wir erstmals die Unterscheidung einer tabuisierten von einer nicht-tabuisierten Diversity ein. Dem üblichen Verständnis ${ }^{9}$ folgend sprechen wir von einer tabuisierten Diversity, wenn über eine bestimmte Unterschiedlichkeit bzw. über einen Teilaspekt dieser Unterschiedlichkeit nicht gesprochen werden darf. Tabuisierung ist eine Frage des Grades. Tabuisierungen dienen in der Regel dazu, das Manifestwerden latent vorhandener Konflikte zu verhindern. ${ }^{10}$ Die Unterscheidung einer tabuisierten von einer nicht-tabuisierten Diversity erscheint $\mathrm{u}$. a. deswegen relevant, weil sich die tabuisierte und die nicht-tabuisierte Diversity konfliktbezogen mit unterschiedlichen Schwierigkeiten verbinden können. Für die Praxis des Managements ist es wichtig, diese Schwierigkeiten zu präzisieren, um Risiken und Gefahren des oben aufgezeigten Trends zu mehr Diversity genauer einschätzen zu können. 
So wie sich z. B. Alter und Geschlecht in einer Organisation unterschiedlich verteilen können, können sich auch religiöse Zugehörigkeiten und Gewerkschaftszugehörigkeiten unterschiedlich verteilen. Derartige Unterschiede werden in der Literatur unter dem Begriff der demografischen Diversity diskutiert." Wir werden in unserer Studie auf die Verteilung der Zugehörigkeit zu Religionsgemeinschaften und Gewerkschaften innerhalb einer Organisation abheben, was unten im Einzelnen präzisiert wird. Unsere zentrale Annahme lautet, dass sich eine nicht-tabuisierte gewerkschaftliche Diversity unter bestimmten Bedingungen mit der Gefahr einer Konflikt-Eskalation, eine tabuisierte religiöse Diversity dagegen mit der Gefahr einer Konflikt-Verschiebung verbindet.

Vorliegende Arbeiten zur Freisetzung von Konflikten im Zuge von Diversity ${ }^{12}$ haben die Dynamik von Tabuisierung/Nicht-Tabuisierung und entsprechend die Dynamik der Konflikteskalation und der Konfliktverschiebung im Kontext demografischer Merkmale bisher nicht diskutiert. Hier liegt die theoriebezogene Erkenntnislücke, die wir mit diesem Beitrag zu schließen versuchen. Wir zeigen zum einen, unter welchen Bedingungen sich in welcher Weise im Zuge von nicht-tabuisierter (demografischer) Diversity Konflikt-Eskalationen zwischen Subgruppen vermitteln können. Wir zeigen zum zweiten, wie tabuisierte Unterschiede und damit latent bleibende Konfliktpotentiale auf nicht-tabuisierte Unterschiede und darin enthaltene Konfliktpotentiale verschoben werden.

Aus unseren Ergebnissen ergeben sich Implikationen für die Praxis des Diversity- bzw. Konfliktmanagements. Werden Konflikt-Verschiebungen als Folge tabuisierter Unterschiede nicht als solche erkannt, so besteht die Gefahr, dass ein Problem zu lösen versucht wird, das gar nicht existiert, während das tatsächliche Problem unerkannt fortexistiert. Ist dem Manager dagegen die Dynamik der Konflikt-Eskalation nicht-tabuisierter Unterschiede vertraut und kennt er die Bedingungen ihres Auftretens, so können sich für ihn Ansatzpunkte für eine Abschwächung der Eskalation ergeben. Auf derartige Konsequenzen für das Management wird in der späteren Diskussion eingegangen.

Die Analyse der differentiellen Effekte tabuisierter versus nicht-tabuisierter Diversity und ihrer jeweils zugrundeliegenden Dynamik bedarf eines geeigneten Untersuchungsfeldes. Auf der Suche nach einem solchen Feld haben wir im Rahmen eines Forschungsprojekts in Schulen in Südindien (Kerala) von Experten Hinweise darauf bekommen, dass die Diskussion der Beziehungen zwischen verschiedenen religiösen Gruppen (hier: Hindus, Moslems und Christen) in Schulen mit einem Tabu belegt ist; dies hängt historisch u. a. mit der Abtrennung Pakistans von Indien im Jahre 1947 zusammen. ${ }^{13}$ Die Frage der Säkularisierung des Bildungswesens, zu der die verschiedenen Lehrergewerkschaften in Kerala eine unterschiedliche Position einnehmen, ist dagegen nicht tabuisiert. Aus diesem Grund stellen wir in dieser explorativen Studie die tabuisierte religiöse Diversity der nicht-tabuisierten gewerkschaftlichen Diversity in ihrer unterschiedlichen konfliktauslösenden Dynamik gegenüber.

Dem üblichen Verständnis in der Forschung zur demografischen Diversity folgend ${ }^{14}$ bezieht sich religiöse Diversity nachstehend auf die Anzahl und Verteilung der in einer Organisation repräsentierten Religionsgemeinschaften (in unserer Studie: Hinduismus, Islam, Christentum); gewerkschaftliche Diversity bezieht sich analog auf die Anzahl und Verteilung der in einer Organisation repräsentierten unterschiedlichen Gewerkschaften. Dabei ist die Diversity umso stärker ausgeprägt, je mehr unterschiedliche Religionsgemeinschaften bzw. Gewerkschaften in einer Schule repräsentiert sind und je mehr sich deren Verteilung einer Gleichverteilung annähert. ${ }^{15}$ Eine zunehmende Diversity geht 
insofern nicht nur mit einer zunehmenden Unterschiedlichkeit der in der Organisation respräsentierten religiösen bzw. politischen Überzeugungen einher, sondern auch mit einer Verschiebung der quantitativen Gewichte der verschiedenen Gruppierungen.

Dieser zuletzt genannte Aspekt wird im Rahmen der Diskussion demografischer Diversity bisher nur unzureichend erörtert. ${ }^{16}$ Hierin sehen wir eine weitere Forschungslücke. Auf den ersten Blick könnte man annehmen, dass sich eine Annäherung an eine Gleichverteilung der verschiedenen Gruppierungen befriedend auswirkt. Diese Annahme mag begründbar sein, wenn man nur die organisationsinternen Verhältnisse betrachtet. Jede Organisation steht jedoch in einem sozialen bzw. kulturellen Kontext. In Indien (und auch in Kerala) bilden z. B. Hindus die stärkste religiöse Gruppierung. ${ }^{17}$ In diesem Kontext kann eine Tendenz zur Gleichverteilung der religiösen Zugehörigkeiten innerhalb einer Organisation von den Hindus als Bedrohung ihrer traditionellen Mehrheit empfunden werden. ${ }^{18}$ Analoges gilt im Hinblick auf die Verteilung der Gewerkschaftszugehörigkeiten. Aus diesem Grund werden wir dem sozio-kulturellen Kontext unserer Studie besondere Aufmerksamkeit widmen.

Eine vergleichende Gegenüberstellung einer weitgehend tabuisierten und einer nichttabuisierten Form demografischer Diversity ist bisher weder in der Diversity-Forschung noch in der Konfliktforschung durchgeführt worden. Dabei interessieren wir uns nicht primär für die konkreten Inhalte bzw. Gegenstandsbereiche, die einer Tabuisierung unterliegen bzw. nicht unterliegen; uns interessiert vielmehr die abstrakte Frage, unter welchen Bedingungen und aufgrund welcher Mechanismen generell eine nicht-tabuisierte (demografische) Diversity mit Konflikt-Eskalation verbunden ist und unter welchen Bedingungen und aufgrund welcher Mechanismen generell eine tabuisierte Diversity mit einer Konfliktverschiebung einhergeht. Daher werden wir versuchen, die (von uns vermuteten) Generalisierungspotentiale für das Management derartiger Diversity sowohl für Kerala als auch für Kontexte außerhalb der indischen Stichprobe sichtbar zu machen.

\section{Voruntersuchung}

Unsere Studie ist als explorative Studie zu verstehen, die den ausgewählten südindischen Kontext - Kerala - als exemplarisches Untersuchungsfeld verwendet. Wir gehen daher nachstehend zunächst knapp auf den Untersuchungskontext unserer Studie und unserer Voruntersuchung ein und nehmen anschließend auf gewerkschafts- und religionsbezogene Besonderheiten in Kerala Bezug.

Wir untersuchen in Kerala die Lehrerkollegien von Schulen, in denen wir die konfliktbezogenen Effekte einer gewerkschaftlichen Diversity und einer religiösen Diversity betrachten. Diese Lehrerkollegien treffen sich regelmäßig einmal in der Woche, um neue pädagogische Instrumente zu entwickeln. Entsprechend den Vorgaben der vorgesetzten Aufsichtsbehörde sollen diese Instrumente den Übergang von einem lehrerzentrierten zu einem schülerzentrierten Lehren und Lernen ermöglichen. Konkret soll durch das Kollegium der Gedanke eines „,collaborative classroom“ präzisiert und implementiert werden. Diese Umsetzung verlangt unter den Lehrern einen intensiven Gedankenaustausch und wechselseitige Hilfestellungen. ${ }^{19}$ 
Um den gesellschaftlichen Kontext unserer Studie adäquat verstehen zu können, haben wir der quantitativen Datenerhebung eine qualitative Vorstudie vorausgeschickt. ${ }^{20}$ Der Erst- und der Drittautor führten zunächst ausführliche nicht-standardisierte ExpertenInterviews in Kerala mit vier indischen Kollegen am Indian Institute of Management Kozhikode (IIMK) durch, in denen der kulturelle und politische Hintergrund von Diversity an sich und von religiöser Diversity im indischen Kontext und im Bundesstaat Kerala diskutiert wurden. Im Mittelpunkt standen sogenannte „kritische Ereignisse“ (z. B. die Ermordung eines Lehrers durch religiöse Extremisten in Kerala $\left.{ }^{21}\right)$. Anhand dieser kritischen Ereignisse versuchten wir, Informationen über die religiösen und politischen Hintergründe von Konflikten speziell im Schulkontext in Kerala in Erfahrung zu bringen. Die sich bei uns herausbildenden Hypothesen wurden anschließend durch Interviews in den Schulen selbst vertiefend erörtert. Es wurden vier Interviews mit Lehrern und zwei Interviews mit Schulleitern vor Ort geführt. Im Vordergrund stand die Frage, wodurch die Zusammenarbeit zwischen den Lehrern in dem obigen Projekt behindert oder auch gefördert wird und inwieweit dabei religiöse und politische Faktoren von Bedeutung sind. Lehrer und Schulleiter wurden gebeten, uns jeweils Beispiele (kritische Ereignisse) $\mathrm{zu}$ benennen und deren Verlauf zu schildern. Für uns ging es dabei wesentlich um die Klärung der Frage, welche Probleme innerhalb der Schulen ohne Schwierigkeiten offen diskutiert werden können und welche Zusammenhänge mit einem Tabu belegt zu sein scheinen. Gelegentlich haben die Interviewten von sich aus berichtet, dass man über bestimmte Dinge in der Schule lieber nicht sprechen sollte (dabei wurde etwa auf Aktivitäten spezieller Gruppierungen - z. B. der Popular Front of India (PFI) oder der Nair Service Society (NSS) ${ }^{22-}$ Bezug genommen). Die Auskunftsbereitschaft erschien uns ausgesprochen hoch. Von Mitgliedern des in Indien und in Kerala hochangesehenen IIMK interviewt zu werden, wurde - so unser Eindruck - als Auszeichnung verstanden. Ausführlich wurde dabei die zentrale Rolle der Lehrergewerkschaften im Rahmen der Auseinandersetzungen um die Säkularisierung der Bildung in Kerala diskutiert. Zusätzlich haben wir vier Interviews mit externen Experten (zwei Moslems, zwei Hindus) geführt, die über detaillierte Kenntnisse des Kontextes unserer Studie verfügen. Diese Experten gehören einer selbstständigen schulunabhängigen Organisation an und besuchen die Schulen regelmäßig im Auftrag der Schulaufsichtsbehörde. Sie unterstützen die Lehrer in Form von Trainings und Beratungen bei der Präzisierung und Umsetzung der Idee eines „collaborative classroom". Auch bei diesen Interviews standen die oben diskutierten Fragen im Vordergrund. Die Interviews wurden in englischer Sprache geführt und dauerten im Durchschnitt etwa 90 Minuten.

Für die Auswertung der Interviews wurden die Eindrücke und Notizen der beiden Interviewer abgeglichen und Unklarheiten in dem jeweils folgenden Interview auszuräumen versucht. Als zentrales Ergebnis der Vorstudie kann zusammengefasst werden, dass sich im Schulbereich in der Tat stärker tabuisierte von nicht-tabuisierten Konfliktfeldern unterscheiden lassen. Das Thema der Säkularisierung der Bildung ist zwar ein hochpolitisches Feld, aber nicht tabuisiert. Regelmäßig stellen Repräsentanten der jeweiligen Lehrergewerkschaft ihr Programm in der Schule vor und diskutieren hierüber anschließend mit den Lehrern. Das Säkularisierungsproblem konkretisiert sich außerdem z. B. in der Ausgestaltung von Lehrbüchern, die von den Lehrern anschließend eingesetzt werden sollen. Diskussionen in der Schule über diese Unterrichtsmaterialien sind insofern gar 
nicht zu vermeiden, so dass das Säkularisierungsproblem auch kaum tabuisierbar ist. Anderes gilt in Bezug auf die Beziehungen zwischen religiösen Gruppierungen. Nichtmuslimische Lehrer berichteten, Moslems würden auch nicht-despektierlich gemeinte Äußerungen über Mohammed schnell als despektierlich interpretieren und sehr emotional reagieren. Würden z. B. Hindu-Lehrer derartige Reaktionsunterschiede zwischen den Religionen öffentlich in der Schule, auch gegenüber ihren muslimischen Lehrerkollegen, artikulieren und zur Diskussion stellen, so wäre dies ein Beispiel für einen (unvorstellbaren) eklatanten Tabubruch.

\section{Gewerkschaftliche Diversity und religiöse Diversity im Kontext}

Sowohl Indien insgesamt als auch speziell der Bundesstaat Kerala zeichnen sich durch Besonderheiten aus, die zum Verständnis unserer Hypothesen und Ergebnisse wichtig sind und deswegen nachstehend knapp beschrieben werden.

\subsection{Gewerkschaftliche Diversity}

Im Bundesstaat Kerala werden 36 Lehrergewerkschaften unterschieden. ${ }^{23}$ Für unseren Untersuchungskontext sind vor allem die folgenden drei Gruppen von Gewerkschaften relevant, die zugleich die größten Mitgliederzahlen aufweisen (alle übrigen in Kerala vorhandenen Lehrergewerkschaften vertreten jeweils weniger als $1 \%$ der Lehrer und werden daher in unserer Studie nicht berücksichtigt): ${ }^{24}$

- Gruppe 1: KSTA (Kerala State Teachers' Association).

- Gruppe 2: KAPTU (Kerala Aided Primary Teachers' Union) und GSTU (Government School Teachers' Union)

- Grupe 3: KTF (Kerala Arabic Teachers' Federation).

Die in unserer Vorstudie befragten Experten haben uns darauf hingewiesen, dass die Unterschiede zwischen den in Gruppe 2 zusammengefassten Gewerkschaften marginal seien und uns daher empfohlen, die Gewerkschaften in der beschriebenen Weise in drei Gruppen zu untergliedern. Die Unterschiede zwischen diesen drei Gewerkschaftsgruppen lassen sich schulbezogen auf der Basis der übereinstimmenden Expertenaussagen vor allem in Bezug auf das Problem der Säkularisierung beschreiben. Gruppe 1 (KSTA) tritt sehr stark für eine säkulare Erziehung ein. Für Gruppe 2 (KAPTU und GSTU) gilt dies weniger nachdrücklich; Gruppe 3 (KTF) plädiert dagegen für eine nicht-säkulare Erziehung. In dieser letzten Gruppe finden sich vergleichsweise viele Moslems.

Diese Unterschiede ergeben sich teilweise aus historischen, teilweise aus gegenwärtigen politischen Zusammenhängen. Neben Westbengalen ist Kerala in Indien der einzige Bundesstaat mit einer kommunistischen Regierung. Die in Kerala regierende Communist Party of India (Marxist) - CPI (M) - setzt sich nachdrücklich für eine säkulare Erziehung an den Schulen ein. Es gilt unter Experten des Erziehungssystems als ausgemacht, dass die zuerst genannte KSTA unter dem Einfluss der marxistischen CPI-M steht. Die Mehrheit der Lehrer an Grund- und Hauptschulen in Kerala gehört der KSTA an. ${ }^{25}$ 
Um das Spannungspotential zwischen den drei Gewerkschaftsgruppen im Kontext der Säkularisierungsfrage zu verstehen, muss man wissen, dass das Schulwesen in Kerala wesentlich von religiösen Gruppierungen begründet wurde. Initiatoren und Träger der Erziehung waren u.a. christliche Missionare, die Nair Service Society und die Muslim Education Society. ${ }^{26}$ Innerhalb der oben genannten Gewerkschaftsgruppe 2 (KAPTU und GSTU) finden sich daher auch viele vor allem christliche Gläubige, die einer Säkularisierung des Bildungswesens eher aus Gründen der in der Verfassung verankerten Gleichheitsidee, aber nicht mit marxistischer Begründung zustimmen. Darüberhinaus haben sich aus den genannten religiösen Gruppierungen einflussreiche Teilgruppen entwickelt, die als christliche oder muslimische Minoritäten auf der politischen Ebene mit besonderem Nachdruck für eine nicht-säkulare Erziehung eintreten. ${ }^{27}$ Die Beziehungen zwischen den drei Gewerkschaftsgruppen weisen damit ein erhebliches Spannungspotential auf, wobei die Konfliktlinie vor allem zwischen der KSTA einerseits und den beiden anderen Gewerkschaftsgruppierungen andererseits verläuft. ${ }^{28}$

Die oben genannte Gruppe 2 (KAPTU und GSTU) wird von der liberal-konservativen „Congress Party“ gelenkt, während die Gruppe 3 (KTF) unter dem Einfluss der „Moslem League" steht. (Die genannten Einflüsse der Parteien auf die Lehrergewerkschaften wurden zum einen von den von uns interviewten Experten und zum zweiten von N.A. Kumar und K.K. George am 25.3.2011 gegenüber dem Drittautor mündlich bestätigt. Die genannten Autoren erklärten zugleich, dass diese Einflussstrukturen ein allgemein geteiltes öffentliches Wissen darstellen, für die jedoch keine offiziellen Quellen angebbar seien).

Dabei unterscheiden sich die Programme der genannten politischen Parteien nicht nur in der Frage der Säkularisierung der Bildung. Die Communist Party India (Marxist) - CPI(M) - plädiert sehr stark, die Congress Party - CP - dagegen weniger stark für staatliche Interventionen, öffentliche Kontrollen und die Verstaatlichung privater Betriebe. Die CPI (M) votiert nachdrücklich gegen die Ausrichtung an der US-Politik und der westlichen Kultur, während die CP eine neutralere Position einnimmt. Die Moslem League kämpft vor allem für die Wahrung der sozialpolitischen Interessen der muslimischen Minderheit und für eine Unterstützung der ca. 10.000 Madressas in Kerala (siehe die Klammerbemerkung im vorausgegangenen Absatz).

Die Experten berichteten uns, dass die Lehrergewerkschaften ein wesentliches Instrument der Parteien darstellen, ihre Politik in der Bevölkerung umzusetzen. Entsprechend kann angenommen werden, dass sich die drei Gruppen von Lehrergewerkschaften und damit auch ihre Mitglieder als Resultat der parteipolitischen Einflussnahme in ihren Überzeugungen nicht nur bezüglich der Säkularisierung unterscheiden. Gelegentlich lassen sich die spezifischen politischen Ausrichtungen der drei Lehrergewerkschaften duch Notizen in der Zeitung belegen. So berichtet, "The Hindu“ am 10.2.2006 über ein Treffen der KSTA- Delegierten, anlässlich dessen der Generalsekretär der KSTA den Kongress mit einem Vortrag „Resist imperialist aggression and prevent commercialisation of education" eröffnet. ${ }^{29}$

Das sich primär an der Säkularisierungsfrage entzündende Konfliktpotential bleibt auch innerhalb einer Schule nicht latent, da sich die diesbezüglichen politischen Vorgaben in Maßnahmen konkretisieren, die innerhalb der Schule Diskussionen unvermeidlich machen: 
- Laut Aussagen der interviewten Experten wurde die von der Regierung 2006 propagierte und von der marxistischen KSTA unterstützte Idee eines ,,collaborative classrooms" von nicht wenigen Lehrern anfänglich als pädagogischer Umsturzversuch mit kommunistisch-atheistischer Zielrichtung gedeutet. Etwa $10 \%$ der Lehrer hätten sich massiv widersetzt. Das staatlich verordnete Programm wurde u. a. mit Überwachungsmaßnahmen und Androhungen von Sanktionen (Kürzungen von Zulagen, Versetzung der Lehrer) durchzusetzen versucht.

- Sehr bekannt geworden ist in Kerala die Schulbuch-Affäre aus dem Jahre 2008. In dem von der marxistischen Regierung lancierten Schulbuch fanden sich Stellungnahmen zugunsten einer freien Wahl der Religion und zu Gunsten einer Heirat zwischen den Religionen und Kasten. Dies führte zu öffentlichen Verbrennungen dieser Schulbücher durch religiöse Extremisten, Streiks von Lehrern an verschiedenen Schulen und zur Ermordung eines Schulleiters. ${ }^{30}$

- Ebenfalls bekannt wurde in Kerala die Auseinandersetzung um ein Lehrbuch zur biologisch-sexuellen Aufklärung, das aus Sicht religiöser Gruppierungen pornografische Bilder beinhaltete. ${ }^{31}$ Der These der Befürworter des Aufklärungsbuches „It teaches just science..." stand die These entgegen „Sex is a sacred thing. It is not simply biological...".32

Inhaltliche Unterschiede zwischen den Gewerkschaften sowie unterschiedliche Majoritätsverhältnisse vermitteln zusammenfassend ein hohes Konfliktpotential, das angesichts strittiger Vorgaben zur Unterrichts- und Lehrmittelgestaltung die Lehrer unmittelbar betrifft und damit auch innerhalb der Schulen manifest wird.

\subsection{Religiöse Diversity}

$80,5 \%$ der indischen Gesamt-Bevölkerung sind Hindus, 13,4 \% sind Moslems und lediglich 2,3\% sind Christen. ${ }^{33}$ Abweichend hiervon sind in Kerala lediglich 56,2 \% Hindus; dagegen finden sich in Kerala 24,7\% Moslems und 19,1 \% Christen. Der hohe Anteil von Moslems und Christen in Kerala steht in Zusammenhang mit der erwähnten Begründung des Erziehungswesens durch religiöse Institutionen und dem in früheren Zeiten regen Handel mit den arabischen Staaten ${ }^{34}$ Auch in Kerala stellt sich auf der Basis dieser Zahlen ein Majoritäten-/Minoritätenproblem, wenngleich aufgrund der quantitativ unterschiedlichen Verteilungen der Religionsgemeinschaften in Kerala vermutlich in anderer Weise als im übrigen Indien. So könnte z. B. der hohe Prozentsatz an Moslems muslimische Gläubige in Kerala politisch ermutigen. In diesem Sinne könnte die Notiz verstanden werden, dass die muslimische Popular Front of India verkündet hat, sie wolle Kerala innerhalb der nächsten 20 Jahre in einen muslimischen Staat transformieren. ${ }^{35}$ Generell wird in Bezug auf Kerala festgestellt, dass religiöse Minoritäten einen unverhältnismäßig großen Einfluss auf das kommunale Geschehen haben. ${ }^{36}$

Spannungen zwischen den religiösen Gruppierungen sind insofern zu erwarten. Eben diese werden aber, so das Ergebnis der Gespräche mit den Experten, tendenziell mit einem Tabu belegt. Tabus können sich auf bestimmte Themenfelder wie z. B. Sexualitä $t^{37}$, Handlungen und Emotionen - z. B. Neid oder Revanchebestrebungen ${ }^{38}-$, aber auch auf Wörter und Gesten ${ }^{39}$ beziehen. Für manche religiöse Bezugsgruppen stellten die Fotos in dem oben erwähnten Aufklärungsbuch einen eklatanten Tabubruch dar. Für Tabus ist 
charakteristisch, dass sie weder schriftlich fixiert noch mündlich expliziert werden, sondern unausgesprochen gelten; ihre Gültigkeit wird stillschweigend vorausgesetzt. Dass im religiösen Bereich mit Tabus zu rechnen ist, wurde uns bereits durch die Empfehlung der Experten signalisiert, in unserer Studie auf keinen Fall von einer religiösen Diversity zu sprechen. Wir könnten bestenfalls nach der Religionszugehörigkeit fragen, aber nicht z. B. nach der Bedeutung der Religionszugehörigkeit für das Selbstverständnis der Befragten. Die prognostizierte Empfindlichkeit bestätigte sich insofern, als sich im Verlaufe unserer quantitativen Datenerhebung einige der befragten Lehrer bei der aufsichtsführenden Schulbehörde darüber beschwerten, dass in unserem Fragebogen Fragen zur Religionszugehörigkeit enthalten waren. Daraufhin wurden wir von der Schulbehörde mündlich und schriftlich angewiesen, im weiteren Fortgang der Untersuchung von derartigen Fragen abzusehen (die Datenerhebung war bereits abgeschlossen).

Existierende religionsbezogene Problemfelder werden mit einem Tabu belegt, um potentielle Widersprüche unkenntlich zu machen..$^{40}$ In den Interviews mit Lehrern und Schulleitern wurde uns z. B. immer wieder versichert, Spannungen zwischen den Angehörigen verschiedener Religionen bestünden nicht auf der privaten, sondern nur auf der politischen Ebene. Man selbst habe z. B. als Hindu nichts gegen Nicht-Hindus. Auf der politischen Ebene würden Spannungen aber in der Tat bestehen, ausgelöst u.a. durch immer wieder vorkommende Attentate religiöser Extremisten, die die Politiker für ihre Zwecke „,ausschlachten“ würden. Diese Aussage ist insofern interessant, als eine solche „Ausschlachtung" erst dann möglich wird, wenn eine diesbezügliche Resonanz hierzu bei den Wählern vorausgesetzt werden kann - religiös motivierte Spannungen also latent vorhanden sind. In diesem Kontext fällt auf, dass einige der Interviewten, die in dieser Weise argumentieren, gleichzeitig eine Partei wählen, die mit nationalistischem Hintergrund Hindus gegenüber Nicht-Hindus zu bevorzugen versucht. Zumindest in dieser Wählergruppe werden Ambivalenzen erkennbar, die nach unserem Eindruck auf latente und tabuisierte Konfliktpotentiale hinweisen.

Je mehr Tabus eine verschleiernde Funktion erfüllen, desto eher kann dort mit Tabus gerechnet werden, wo im religiösen Kontext Anspruch und Realität in Bezug auf die Beziehungen zwischen den Religionen deutlich auseinanderklaffen. Wir geben hierzu im Kontext von Indien und Kerala einige weitere in der Literatur dokumentierte Beispiele:

- Nicht Diversity, sondern Hindu-Sein ist Kern der nationalen und religiösen Identität in Indien. ${ }^{41}$ Damit ist auch das Kastenwesen integraler Bestandteil der religiösen Identität. ${ }^{42}$ Gleichzeitig bekämpft die Regierung das Jahrhunderte alte identitätsstiftende Kastenwesen mit Nachdruck. ${ }^{43}$ Dieser Kampf und entsprechende soziale Programme werden aber ihrerseits von konservativen religiösen Hindu-Gruppierungen in Indien als Ausdruck einer ,De-Sanskritisation" kritisiert", womit sich gravierende Widersprüiche ergeben.

- Der Kampf gegen das Kastenwesen wird mit besonderem Nachdruck in Kerala geführt. Dies ändert aber nichts daran, dass auch in Kerala die unterprivilegierten Kasten und Ethnien in den Schulen unterrepräsentiert sind ${ }^{45}$ und es in Kerala religiöse Gruppierungen gibt, die - abweichend z. B. von manchen christlichen Gruppierungen - für eine Beibehaltung des Kastenwesens votieren. ${ }^{46}$ 
- Auf der nationalen Ebene hat sich in Indien - vermutlich auch als Reaktion auf die Schrecknisse der Abtrennung Pakistans von Indien 1947 und die von der Elterngeneration noch erinnerten Gewalttaten - auf der gesellschaftlichen Ebene eine soziale Norm entwickelt, die in der Kurz-Formel „Unity in Diversity“ zusammengefasst wird. Gleichzeitig liegen aber Hinweise auf religiöse Vormachtsansprüche auf Seiten der Hindus vor ${ }^{47}$, die auf der politischen Ebene von einer bestimmten Partei (BJP) speziell nach dem Mumbai-Attentat 2008 auch offen artikuliert wurden. Die Wiederbelebung einer religiös-nationalen Hinduismusbewegung ${ }^{48}$ steht im Übrigen im Widerspruch zum offiziellen Gleichheitspostulat. Sie verschärft nicht nur die Spannungen zwischen Hindus und Moslems ${ }^{49}$, sondern auch die Spannungen zwischen Hindus und Christen, die ihrerseits in der Vergangenheit u.a. durch Missionierungsversuche von Christen gegenüber Hindus intensiviert wurden und in blutige Auseinandersetzungen mündeten. ${ }^{50}$

- Auch in Kerala bestehen, was die Beziehungen zwischen den Religionen anbelangt, deutliche Differenzen zwischen Anspruch und Realität. Der Anspruch konkretisiert sich in den Leitideen einer (kommunalen) Harmonie und eines hohes Bildungsgrades. ${ }^{51}$ Der hohe Bildungsgrad bestätigt sich in Kerala insofern, als die Analphabetenrate in Kerala mit weniger als $5 \%$ sehr niedrig ist ${ }^{52}$ (die Analphabetenrate beträgt im gesamten Indien im Census $201126 \%)^{53}$. Kerala ist stolz auf die auch im ländlichen Bereich intensive Beschulung. ${ }^{54}$ Gerade weil Bildung ein Ideal darstellt, wurde der Vorfall, bei dem ein muslimischer Extremist den Finger eines hinduistischen Lehrers abschnitt, in der Öffentlichkeit als Rückfall in die Barbarei und als Beflecken der Kerala-Ideale einer aufgeklärten Gesellschaft gebrandmarkt. ${ }^{55}$ Dabei ist aber gleichzeitig zu berücksichtigen, dass in Kerala im Mai 2002 im Fischerdorf Marad muslimische Fischer von Hindu-Fischern grausam ermordet wurden. ${ }^{56}$

Unser Fazit: Je mehr Anspruch und Realität - gemessen an den Kriterien Harmonie und Bildung - auseinanderklaffen, desto eher wird diese Differenz tabuisiert. Je mehr diese Differenz tabuisiert wird, Konfliktpotentiale im religiösen Bereich also unterdrückt werden, desto eruptiver und ungestörter brechen sie sich gegebenenfalls eine Bahn. ${ }^{57}$ Der Streit um Fischerei-Rechte wird zum geeigneten Medium, um im Sinne einer Konfliktverschiebung tabuisierte religiöse Spannungen auszutragen.

\section{Gewerkschaftliche Diversity, religiöse Diversity und Konflikte - Theorie und Hypothesen}

\subsection{Gewerkschaftliche Diversity und Konflikte}

Theoriebezogen ergeben sich aus den Beschreibungen der gewerkschaftlichen Diversity und ihrer Hintergründe folgende Implikationen: Die unvermeidlichen Diskussionen über die Unterrichtsmittel machen die unterschiedlichen Auffassungen der jeweiligen Gewerkschaftsgruppierungen sichtbar. Es ergibt sich demnach ein aufgabenbezogener Dissens. ${ }^{58}$ Je mehr die Auffassungsunterschiede, die mit spezifischen Werthaltungen parallelgehen (s. o.), für das Selbstverständnis der Angehörigen der jeweiligen Gewerkschaften von 
Bedeutung sind, desto eher gewinnt die Kategorie Gewerkschaftszugehörigkeit an Relevanz $^{59}$ und desto größer ist die Gefahr, dass die wahrgenommenen Unterschiede über Prozesse der sozialen Kategorisierung ${ }^{60}$ in Subgruppen-Bildungen im Kollegium und emotionale Konflikte zwischen diesen Subgruppen einmünden. Daher gehen wir von einer positiven Beziehung zwischen gewerkschaftlicher Diversity einerseits und der Freisetzung gruppeninterner Konflikte andererseits aus.

Diese gruppeninternen Konflikte werden durch Prozesse auf der dyadischen Ebene zusätzlich verstärkt: Da kritisches Hinterfragen der Position des Anderen im Zuge von Diskussionen nicht selten persönlich genommen wird ${ }^{61}$, können sich Beziehungskonflikte $^{62}$ entwickeln, die durch emotionale Spannungen zwischen Personen definiert sind. Das direkte Austragen dieser Spannungen birgt für die Beteiligten erhebliche Verletzungsrisiken. ${ }^{63} \mathrm{Je}$ mehr es über soziale Kategorisierungen zu Subgruppenbildungen und Spannungen zwischen diesen Subgruppen kommt, desto eher werden - so unsere Annahme - emotionale Spannungen zwischen einzelnen Personen auf die sich entwickelnden Spannungen zwischen den Subgruppen umgelenkt bzw. übertragen. Der Konflikt wird gleichsam auf eine andere Aggregatebene verschoben. Geschützt durch seine Subgruppe kann der Einzelne persönliche Beziehungskonflikte mit einem Mitglied der gegnerischen Subgruppe risikofreier und indirekt - unter dem Deckmantel subgruppenspezifischer Konfliktfelder - austragen. Subgruppenspannungen werden auf diesem Wege zusätzlich intensiviert. Damit kann eine sich selbst verstärkende Dynamik freigesetzt werden, die ein Eskalationspotential enthält. ${ }^{64}$ Wir vermuten daher, dass die positive Beziehung zwischen gewerkschaftlicher Diversity und gruppeninternen Konflikten positiv beschleunigt anwächst.

Die Tendenz zur Konflikt-Eskalation wird darïber hinaus durch die mehrfach angesprochene Majoritäten-/Minoritäten-Konstellation verstärkt. Der quantitativ dominierenden marxistischen Gewerkschaft kann von den Minoritäten im Kontext der kommunistischen Regierung in Kerala ein Vormachtsanspruch zugeschrieben werden. Diese Zuschreibung ist schon deswegen plausibel, weil Mehrheiten bekanntermaßen - konfrontiert mit Diversity - nicht zu einer Strategie des Multikulturalismus (vgl. oben „Unity in Diversity“), sondern zur einer Unterordnung verlangenden Assimilationsstrategie tendieren. ${ }^{65}$ Im hier untersuchten Kontext ist der Vormachtsanspruch der marxistischen Gewerkschaft überdies durch den erwähnten Einsatz von Sanktionen durch die Regierung objektiv belegt. Dies verbindet sich für die Minoritäten-Gewerkschaften mit einer Bedrohung ihrer sozialen und professionellen Identität ${ }^{60}$, auf die sie mit forcierten und artikulierten Gleichwertigkeits-Ansprüchen reagieren. Dies kann für die quantitativ dominierende Gewerkschaft wiederum zur Bedrohung werden, die dann ihrerseits forciert reagiert, so dass der Konflikt eskaliert. Die Konflikt-Eskalation vermittelt sich demnach auf mehreren Aggregatebenen. Neben intraorganisationalen dyadischen Prozessen sind dabei politisch mitbedingte Mehrheiten-/Minderheiten-Konstellationen bedeutsam.

Hypothese 1: Die Beziehung zwischen gewerkschaftlicher Diversity und gruppeninternen Konflikten hat einen positiv beschleunigten Verlauf. 


\subsection{Religiöse Diversity und Konflikte}

Für Religionen ist zum einen kennzeichnend, dass sie für viele Gläubige eine hohe persönliche Relevanz aufweisen. ${ }^{67}$ Zum zweiten ist für Religionen ein hoher Universalitätsanspruch charakteristisch. Religiöse Regeln beziehen sich auf nahezu alle Bereiche des Lebens. ${ }^{68}$ Die Parallelität von Relevanz und Universalitätsanspruch lässt erwarten, dass mit zunehmender Unterschiedlichkeit der in der Gruppe repräsentierten Religionen das (latente) Konfliktpotential steigt. Dies gilt generell und zusätzlich in dem Maße, in dem im sozialen Kontext (hier: Kerala) ausgeprägte Differenzen zwischen dem Ideal eines aufgeklärten Miteinanders verschiedener Religionen einerseits und der Realität andererseits bestehen (s. o.), die zum Zweck der Konfliktvermeidung mit einem Tabu belegt werden. Da die Lehrer in dieser Studie zugleich Bürger Keralas sind, erhöht sich durch ihr Wissen dieser Differenzen das Konfliktpotential und damit die potentielle Bedrohung ihrer religiösen Gruppen-Identität. ${ }^{69}$ Alle drei Religionen wissen um Vormachtsansprüche bestimmter hinduistischer Gruppierungen im übrigen Indien (s. o.), aber auch um Dominanzansprüche muslimischer Extremisten in Kerala (s. o.) und um die politische Sensibilität der quantitativen Verhältnisse der drei Religionsgruppen untereinander. Religionsbezogene Auseinandersetzungen zwischen den Gruppen werden von daher nicht nur gemieden, sondern in Kenntnis der möglichen Eruptionen und deren Antizipation auch in der Schule tabuisiert. Diese Tendenz wird durch das implizite Wissen um die Notwendigkeit einer gedeihlichen Zusammenarbeit in der Organisation zwischen allen religiösen Gruppierungen verstärkt. Diskutiert werden religionsbezogene Fragen entsprechend nur dann, wenn es im Rahmen der Aufgabenerfüllung unvermeidlich ist, wenn z. B. zu entscheiden ist, ob man in Anpassung an hinduistische Überzeugungen auch Astrologie oder unter Berücksichtigung christlicher und muslimischer Überzeugungen nur Astronomie in den Lehrplan aufnehmen soll.

Um das Manifestwerden latenter Spannungen zu vermeiden, bedient man sich neben der Tabuisierung zusätzlich der in vielen Kulturen praktizierten Strategie der Segregation. ${ }^{70}$ Man erklärt Religion zur Privatsache, die vom beruflichen Kontext zu trennen ist. ${ }^{71}$ Insofern ist das Auftreten manifester Konflikte zwischen den religiösen Gruppen trotz vorhandener Konfliktpotentiale innerhalb der Schule nicht zu erwarten.

Die Parallelität von Tabuisierung und Segregation verhindert aber nicht, dass auf der Gruppenebene gegenüber den jeweils anderen Gruppen weiterhin Ressentiments bestehen; dies kann zu dem paradoxen Effekt führen, dass gerade durch die Tabuisierung und Segregation latent vorhandene Spannungen verstärkt werden. Durch das Ausbleiben diesbezüglicher Diskussionen zwischen den Gruppen werden zum einen vorhandene Gemeinsamkeiten zwischen den Religionen nicht erkennbar. Zum zweiten sind gerade bei ausbleibender Diskussion der willkürlichen Zuschreibung nicht vorhandener Unterschiede Tür und Tor geöffnet (so dass Moslems z. B. in die Nähe von Taliban gerückt werden $^{72} ; \mathrm{vgl}$. ähnliche Beobachtungen in den $\mathrm{USA}^{73}$ ). Durch Tabuisierung und Segregation werden die innerhalb der jeweiligen Gruppe attribuierten Differenzen zwischen den Religionen also unter Umständen nicht kleiner, sondern größer. Es ist zu bezweifeln, dass derartige Prozesse und damit einhergehende Spannungspotentiale zwischen den Gruppen unwirksam bleiben. Wir vermuten vielmehr eine Verschiebung der (durch Tabuisierung und Segregation u. U. vergrößerten) latenten Konfliktpotentiale und der in ihnen enthal- 
tenen Energien auf diejenigen Konfliktfelder, deren Austragen für legitim erachtet wird. Die Strategie der Verschiebung erfüllt eine Entlastungsfunktion in genau dem Umfang, in dem die Strategie der Tabuisierung (und Segregation) vorhandene Spannungen stabilisiert oder sogar vergrößert.

Daher nehmen wir an, dass die Beziehung zwischen der nicht tabuisierten gewerkschaftlichen Diversity und dem Auftreten von gruppeninternen Konflikten enger ist, wenn die tabuisierte religiöse Diversity hoch ausgeprägt ist.

Hypothese 2: Religiöse Diversity moderiert die positive Beziehung zwischen der gewerkschaftlichen Diversity und gruppeninternen Konflikten derart, dass diese Beziehung enger ist, wenn die religiöse Diversity hoch ausgeprägt ist.

\section{Hauptuntersuchung}

\section{I Stichprobe und Datenerhebung}

Wir kontaktierten das Office of the Director of Public Instruction im Bundesstaat Kerala, vom dem wir eine Liste von $\mathrm{N}=237$ Grund- und Hauptschulen in allen Regionen Keralas erhielten. Um das Lehrerkollegium jeweils als Gruppe ansprechen zu können, haben wir nur solche Schulen in die Stichprobe aufgenommen, die neben dem Schulleiter zwischen 3 und 30 Lehrer beschäftigen. Der standardisierte Fragebogen wurde - versehen mit einem frankierten Rückcouvert und einem Empfehlungsschreiben des Director of Public Instruction - postalisch an die Schulleiter verschickt. Die Schulleiter wurden gebeten, die Fragebögen in der wöchentlichen Lehrerkonferenz zur Beantwortung auszuhändigen. Außer dem Empfehlungsschreiben des Director of Public Instruction lag jedem Fragebogen ein von uns verfasstes Schreiben bei, in dem der Zweck der Untersuchung - die Aktivierung von Kooperationspotentialen im Lehrerkollegium - beschrieben und Anonymität der Auswertung durch das IIMK zugesichert wurden. Um eine ausreichende Reliabilität unserer Untersuchung sicherzustellen, haben wir nur diejenigen Schulen in unsere endgültige Stichprobe aufgenommen, in denen mindestens $80 \%$ der Lehrerschaft den Fragebogen ausgefüllt zurückgegeben hatten. Die endgültige Stichprobe reduzierte sich dadurch auf $\mathrm{N}=96$ Schulen. Diese Schulen beschäftigten im Durchschnitt 10,4 Lehrer. Das Durchschnittsalter der befragten Lehrer betrug 40 Jahre, die durchschnittliche Beschäftigungsdauer an derselben Schule 9 Jahre; $76 \%$ der Befragten sind weiblich. Die Mehrzahl der befragten Lehrer sind Hindus (56\%), $26 \%$ Christen, $15 \%$ Moslems, und 1,6\% gehören anderen religiösen Gruppierungen an. 1,4\% der Befragten kreuzten die Kategorie „Keine Religion“ an. Für die Verteilung der drei Gewerkschaftsgruppierungen gilt das folgende: 1) Marxistische KSTA=54\%; 2) KAPTU $=12 \%$; GSTU $=10 \%$; 3) $\mathrm{KATF}=4 \%$. Die Frage nach der Religionszugehörigkeit wurde von 98,3\% der Befragten beantwortet, die Frage nach der Gewerkschaftszugehörigkeit von 95,1%. 


\subsection{Operationalisierungen}

Die religiöse Diversity bezüglich der drei Religionsgruppen (Hindus, Moslems, Christen) wurde mit dem Blau-Index ${ }^{74}$ ermittelt. Dieser Wert kann theoretisch Werte zwischen 0 und 1 annehmen. Hohe Werte indizieren, dass alle drei Religionsgruppen gleichmäßig in dem jeweiligen Lehrerkollegium vertreten sind. Die gewerkschaftliche Diversity wurde ebenfalls mit dem Blau-Index erhoben.

Für die Operationalisierung der gruppeninternen Konflikte wurden drei Items von $J h^{75}{ }^{75}$ sowie Jehn und Mannix ${ }^{76}$ adaptiert. 1) „Innerhalb unseres Lehrerkollegiums gibt es verschiedene Gruppierungen, die nicht gut miteinander auskommen“, 2) „In unserem Lehrerkollegium gibt es Spannungen zwischen verschiedenen Gruppierungen", 3) „In unserem Lehrerkollegium werden manchmal emotionale Spannungen zwischen bestimmten Gruppierungen sichtbar". Der Fragebogen wurde vom englischen ins südindische Malayalam übersetzt und rückübersetzt. ${ }^{77}$ Die Reliabilität dieser Skala war mit Cronbach's alpha $=0.89$ ausreichend. Eine Hauptkomponentenanalyse ergab einen Faktor mit einem Eigenwert größer 1, der $83 \%$ der Varianz erklärt. Der James-Koeffizient in Höhe von $r_{W G(J)}=0.91^{78}$ und die Interrater-Reliabilitäten (ICC1 $=0.35$; ICC2 $=0.87$ ) rechtfertigen die Aggregation der individuellen Daten auf dem Level der Lehrerkollegien. ${ }^{79}$

\subsection{Kontrollvariablen}

Als Kontrollvariablen wurden die Schulgröße (operationalisiert über die Anzahl der Lehrer) und drei weitere Diversity-Arten aufgenommen, die das Auftreten von gruppeninternen Konflikten begünstigen können. Die Größe der jeweiligen Schule ist möglicherweise negativ mit der Kohäsion und der Kommunikation in den jeweiligen Kollegien verbunden. ${ }^{80}$ Altersbezogene Diversity, geschlechterbezogene Diversity und die Diversity in Bezug auf die Dauer der Zugehörigkeit zur Schule sind in empirischen Studien als konfliktauslösend bestätigt worden. ${ }^{81}$ Die Alters-Diversity und die Diversity bezüglich der Dauer der Zugehörigkeit zur Schule wurden anhand der Standardabweichung operationalisiert. Die geschlechtsbezogene Diversity wurde dagegen mit dem Blau-Index erfasst. ${ }^{82}$

\section{Ergebnisse}

Mittelwerte, Standardabweichungen und Korrelationen der Variablen sind in Tab. I zusammengefasst.

Nur die Größe der Schule ist signifikant positiv mit gruppeninternen Konflikten verbunden $(r=0.31 ; p<0.01)$. Religiöse Diversity ist mit gruppeninternen Konflikten nicht signifikant verbunden ( $r=0.15$; ns); Gewerkschaftliche Diversity ist auf dem $10 \%$-Niveau signifikant positiv mit gruppeninternen Konflikten verbunden $(r=0.20 ; p<0.10)$.

Unsere Hypothesen wurden anhand einer hierarchischen Regressionsanalsye überprüft (vgl. Tab. 2). Eine Überprüfung der Variance Inflation Factors für die untersuchten unabhängigen Variablen ergab keine Hinweise auf Multikollinearität ${ }^{83}$. Die Kontrollvariablen Schulgröße, geschlechtsbezogene Diversity, Diversity bezüglich der Schulzugehörigkeitsdauer und Alters-Diversity erklären zusammen $11 \%$ der Varianz (vgl. Schritt 
Tab. 1: Arithmetisches Mittel, Standardabweichung und Korrelationen

\begin{tabular}{lrlllllll}
\hline Variable & M & SD & 1 & 2 & 3 & 4 & 5 & 6 \\
\hline 1. Schulgröße & 10.74 & 5.94 & & & & & & \\
2. Geschlechts-Diversity & 0.29 & 0.23 & $0.18^{*}$ & & & & & \\
$\begin{array}{l}\text { 3. Schulzugehörigkeits- } \\
\text { dauer:Diversity }\end{array}$ & 5.85 & 2.72 & $0.17 *$ & 0.01 & & & & \\
4. Alters- Diversity & 7.02 & 2.51 & -0.02 & $0.19 *$ & $0.26 * * *$ & & & \\
5. Gewerkschaftl.Diversity & 0.55 & 0.31 & $0.39 * * *$ & $0.25 * *$ & -0.05 & 0.00 & & \\
6. Religiöse-Diversity & 0.37 & 0.22 & 0.16 & $0.30 * * *$ & -0.11 & 0.08 & $0.34^{* * *}$ & \\
7. Konflikt & 1.63 & 0.44 & $0.31 * * *$ & 0.10 & 0.06 & 0.11 & $0.20 *$ & 0.15 \\
\hline $\mathrm{N}=96$ Schulen; $* \mathrm{p}<0.10, * * \mathrm{p}<0.05, * * * \mathrm{p}<0.01$ & & & & &
\end{tabular}

Tab. 2: Ergebnisse der Regressionsanalyse - abhängige Variable: gruppeninterne Konflikte

\begin{tabular}{|c|c|c|c|c|c|c|}
\hline \multirow[t]{2}{*}{ Unabhängige Variable } & \multicolumn{3}{|l|}{$\mathrm{H} 1$} & \multicolumn{2}{|l|}{$\mathrm{H} 2$} & \multirow{2}{*}{$\begin{array}{l}\mathrm{H} 3 \\
\text { Schritt } \\
6\end{array}$} \\
\hline & $\begin{array}{l}\text { Schritt } \\
1\end{array}$ & $\begin{array}{l}\text { Schritt } \\
2\end{array}$ & $\begin{array}{l}\text { Schritt } \\
3\end{array}$ & $\begin{array}{l}\text { Schritt } \\
4\end{array}$ & $\begin{array}{l}\text { Schritt } \\
5\end{array}$ & \\
\hline \multicolumn{7}{|l|}{ Kontrollvariablen } \\
\hline Schulgröße & $0.29 * * *$ & $0.23 * *$ & $0.29 * * *$ & $0.26 * *$ & $0.22 * *$ & $0.21 * *$ \\
\hline Geschlechts-Diversity & 0.08 & 0.09 & 0.01 & 0.06 & 0.08 & 0.09 \\
\hline $\begin{array}{l}\text { Schulzugehörigkeits- } \\
\text { dauer- Diversity }\end{array}$ & -0.03 & -0.01 & -0.03 & 0.00 & 0.00 & 0.02 \\
\hline Alters-Diversity & 0.10 & 0.10 & 0.10 & 0.09 & 0.09 & 0.06 \\
\hline \multicolumn{7}{|l|}{ Haupteffekte } \\
\hline Gewerkschaftl. Diversity & & $0.20^{* *}$ & $-0.56^{*}$ & & $0.18^{*}$ & $0.24 * *$ \\
\hline $\begin{array}{l}\text { Gewerkschaftl. Diversity } \\
\text { quadriert }\end{array}$ & & & $0.79 * *$ & & & \\
\hline Religiöse Diversity & & & & 0.14 & 0.09 & 0.10 \\
\hline \multicolumn{7}{|l|}{ Interaktionseffekt } \\
\hline $\begin{array}{l}\text { Gewerkschaftl-Diversity } \times \\
\text { religiöse Diversity }\end{array}$ & & & & & & $0.26^{*}$ \\
\hline $\mathrm{R}^{2}$ & 11 & 15 & 20 & 13 & 15.5 & 21 \\
\hline F Werte & 2.831 & 3.146 & 3.692 & 2.651 & 2.732 & 3.502 \\
\hline
\end{tabular}

$\mathrm{N}=96$ Schulen; standardisierte B-Koeffizienten; * $\mathrm{p}<0.10$, ** $\mathrm{p}<0.05, * * \mathrm{p}<0.01$

1). Darüber hinaus zeigt sich in Schritt 2 ein signifikanter Haupteffekt der gewerkschaftlichen Diversity, $\beta=.20 ; p<0.05 ; \Delta R^{2}=4 \% ; F=3.15 ; p<0.05$. Um Hypothese 1 zu prüfen, wurde in Schritt 3 der quadrierte Term für die gewerkschaftliche Diversity in die 
Abb. 1: Gewerkschaftliche Diversity, gewerkschaftliche Diversity quadriert und Konflikt

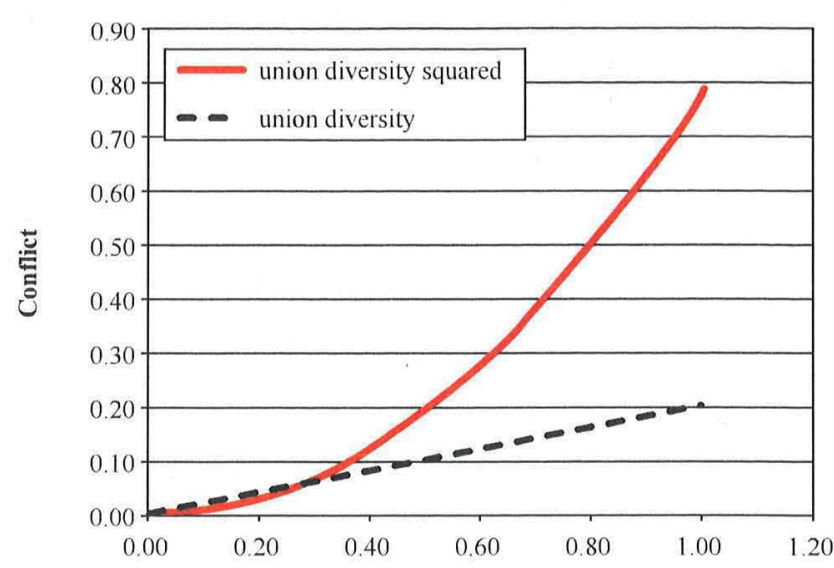

Gleichung aufgenommen. Der $\beta$-Wert für diesen Term wird ebenfalls signifikant, $\beta=0.79$; $p<0.05 ; \Delta R^{2}=5 \% ; F=3.69 ; p<0.05 ;$ die Aufnahme des quadrierten Terms führt zu einer signifikanten Steigerung der aufgeklärten Varianz. Hypothese 1 ist damit bestätigt. Die kurvilineare Beziehung zwischen gewerkschaftlicher Diversity und gruppeninternen Konflikten ist in Abb. 1 dargestellt. Zum Vergleich haben wir in dieser Grafik auch die lineare Funktion zwischen gewerkschaftlicher Diversity und gruppeninternen Konflikten eingezeichnet.

Schritt 4 zeigt, dass die religiöse Diversity erwartungsgemäß nicht signifikant mit gruppeninternen Konflikten verbunden ist, $B=0.14 ; p=0.19$. In Schritt 5 und 6 überprüfen wir den Moderationseffekt der religiösen Diversity auf den Zusammenhang zwischen gewerkschaftlicher Diversity und gruppeninternen Konflikten (Hypothese 2). Es ergibt sich ein signifikanter Interaktionseffekt, $B=0.26 ; p<0.01 ; \Delta R^{2}=5,5 \% ; F=3.50 ; p<0.01$. Abbildung 2 zeigt, dass die Beziehung zwischen gewerkschaftlicher Diversity und gruppeninternen Konflikten enger ist, wenn die religiöse Diversity hoch ausgeprägt ist. Damit ist auch Hypothese 2 bestätigt.

\section{Diskussion}

Angesichts einer zunehmenden demografischen Diversity in Organisationen halten wir es für angemessen, das Management über Risiken einer steigenden Diversity zu informieren. Unter dem Blickwinkel dieser Risiken sind wir von der Annahme ausgegangen, dass es sinnvoll sein könnte, neben den in der Literatur bisher unterschiedenen Formen der Diversity zusätzlich eine tabuisierte von einer nicht-tabuisierten Diversity zu unterscheiden. Unsere leitende These lautete, dass sich Tabuisierungen von Konfliktfeldern mit einer Konfliktverschiebung, Nicht-Tabuisierungen von Konfliktfeldern dagegen mit der Gefahr von Konflikt-Eskalationen verbinden könnten. Die Möglichkeit, dass sich Tabuisierung und Nicht-Tabuisierung von Diversity in unterschiedlicher Weise in Konflikte vermitteln, ist in einschlägigen Theorien zur Dynamik von Diversity bisher nicht berücksichtigt worden. ${ }^{84}$ Des Weiteren wurde bisher nicht genügend berücksichtigt, dass unterschiedliche Grade demografischer Diversity mit der Induktion von Majoritäts- 
Abb. 2: Religiöse Diversity als Moderator des Zusammenhangs zwischen gewerkschaftlicher Diversity und Konflikt

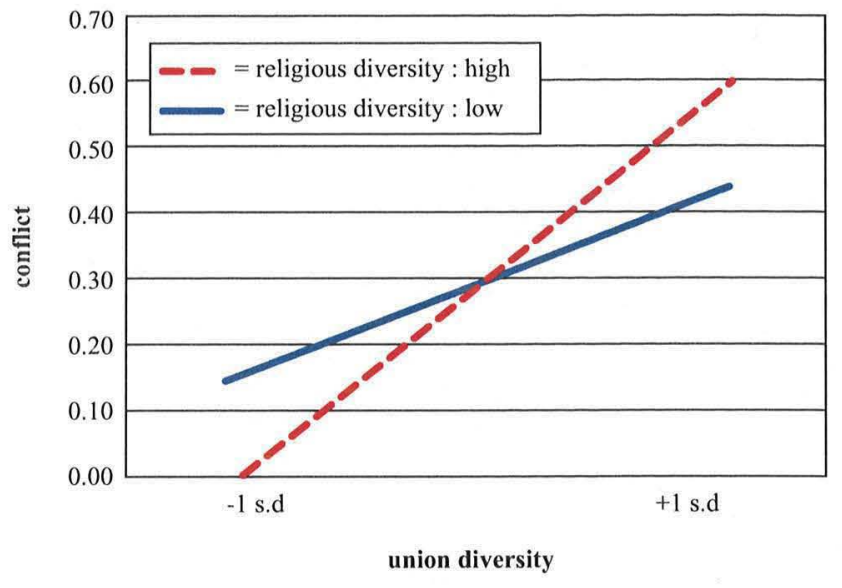

Minoritätsproblemen verbunden sein können (s. o.). Im Zuge eines Forschungsprojektes in Südindien -Kerala- fand sich die Gelegenheit, an einer Stichprobe von Schulen zur Schließung dieser Forschungslücken einen Beitrag zu leisten.

Auf der Basis von Expertenaussagen, unterstützt durch eigene Beobachtungen und die Dokumentation spezifischer Vorkommnisse in Kerala in der Literatur, konnte die These fundiert werden, dass die Beziehungen zwischen den drei Religionsgruppen (Hindus, Christen, Moslems) in Kerala durch hohe latente Spannungspotentiale gekennzeichnet sind, deren explizite Diskussion sowohl außerhalb als auch innerhalb der Schulen jedoch tabuisiert ist. Analog konnte die These gestützt werden, dass die Beziehungen zwischen marxistischen und nicht-marxistischen Lehrergewerkschaften in Kerala außerhalb und innerhalb der Schulen durch einen offenen Disput gekennzeichnet ist; inhaltlich steht dabei die Frage der Säkularisierung des Bildungswesens zur Diskussion, die sich anlässlich spezifischer Vorgaben der kommunistischen Regierung zur Unterrichtsgestaltung zwischen den verschiedenen Lehrergewerkschaften entzündet.

Empirisch zeigte sich, dass die gewerkschaftliche Diversity innerhalb der Schulen mit dem Auftreten von Konflikten positiv verbunden ist (vgl. Tab. 2). Angesichts des Umstandes, dass die Lehrer ihrem Kollegium im Durchschnitt bereits seit 9 Jahren angehören, ist bereits dieser Befund bemerkenswert. In einer gesonderten Regressionsanalyse ergab sich, dass die Dauer der Zugehörigkeit zum Kollegium nicht signifikant mit dem Auftreten von Konflikten verbunden ist, $\beta=-0.18 ; \mathrm{t}=-1.4 ; \mathrm{p}=-0.17$. Auch nach vielen Jahren der Zugehörigkeit zu derselben Schule ist die positive Beziehung zwischen gewerkschaftlicher Diversity und dem Auftreten von Konflikten - abweichend von den Befunden bei Harrison et al. ${ }^{85}$ also nicht abgeschwächt. Dies mag damit zusammenhängen, dass durch die unterrichtsbezogenen Vorgaben der Regierung immer wieder erneut Konflikte zwischen den Gewerkschaftsgruppen innerhalb der Schule induziert werden.

Des Weiteren ließ sich zeigen, dass die gewerkschaftliche Diversity kurvilinear positiv beschleunigt mit Spannungen zwischen Subgruppen innerhalb der Schulen verbunden ist (vgl. Abb. 1). Wir interpretieren diese Kurvilinearität als Widerspiegelung einer KonfliktEskalation zwischen den beteiligten Gruppen. In Bezug auf die religiöse Diversity zeigte sich kein signifikanter Haupteffekt (vgl. Tab. 2) - religiöse Diversity und Konflikt sind 
demnach unverbunden - wohl aber ein Moderationseffekt (vgl. Abb. 2): Die Beziehung zwischen der gewerkschaftlichen Diversity und dem Auftreten von Konflikten ist dann enger, wenn zugleich die religiöse Diversity in der Schule hochausgeprägt ist. Wir haben diesen Befund als Indiz für eine Konfliktverschiebung tabuisierter auf nicht-tabuisierte Konfliktfelder gedeutet.

\subsection{Limitationen und Implikationen für die Forschung}

Interpretiert man die Tabuisierung von Konfliktpotentialen als eine Form des Konfliktmanagements, so deuten unsere Befunde darauf hin, dass diese Strategie zweischneidig ist. Am Beispiel der religiösen Diversity illustriert, scheint sich die Tabuisierung zwar mit dem Vorteil zu verbinden, dass das latente Konfliktpotential in der jeweiligen Konfliktzone nicht manifest wird. Diesen Vorteil scheint man aber mit dem Nachteil zu erkaufen, dass das latente Konfliktpotential in eine inhaltlich andere, nicht-tabuisierte Konfliktzone verschoben wird. Da wir den Mechanismus der Konfliktverschiebung selbst nicht überprüft haben, ist dies jedoch nur eine Hypothese, die einer gesonderten Überprüfung bedarf. Hierzu müssten, wie z. B. Gersick diskutiert ${ }^{86}$, Gruppenprozesse im Längsschnitt analysiert werden. Wir halten es für sinnvoll, in zukünftiger Forschung diese Dynamik auf dem Gruppenniveau näher zu beleuchten. Dabei dürfte es zweckmäßig sein, neben der Verteilung der Religionszugehörigkeiten die Intensität der Religiosität in den religiösen Gruppen mitzuerheben, da das latente Konfliktpotential auch hiervon abhängen wird. (In der vorliegenden Studie hatten uns die Experten von einer entsprechenden Erhebung allerdings abgeraten.)

Analoges gilt in Bezug auf die Prozesse, über die sich eine nicht-tabuisierte Konfliktzone in gruppeninterne Konflikte übersetzt; auch diese Konstellation scheint sich als ambivalent zu erweisen. Als Vorteil ist in Rechnung zu stellen, dass ein manifest gewordener Konflikt eher einer Bearbeitung zugänglich ist; dies gilt zumindest, solange der Konflikt primär einen Aufgabenbezug hat ${ }^{87}$ Als Nachteil deuten die Befunde der Abb. I jedoch auf Risiken einer Konflikteskalierung hin. Eben weil die Diskussion der mit der gewerkschaftlichen Diversity einhergehenden Auffassungsunterschiede keinem Tabu unterliegt, wird sie offener geführt und macht Divergenzen sichtbar. Im Zuge dieser Diskussion können jedoch zum einen Beziehungskonflikte zwischen Personen freigesetzt werden $^{88}$, die nur schwer lösbar sind ${ }^{89}$, gleichzeitig aber das Energiepotential enthalten, das eine Eskalierung der Konflikte zwischen den Subgruppen begünstigt. Eine zunehmende Diversity impliziert zum zweiten definitionsgemäß (siehe Einleitung) eine spezifische Verteilung der quantitativen Gewichte innerhalb der je untersuchten Einheit. Hat eine der Gruppen außerhalb der untersuchten Einheit einen Majoritätenstatus - wie in unserer Studie die marxistische Gewerkschaft KSTA -, so können die quantitativen Verteilungen innerhalb der untersuchten Einheit, die für die Fokal-Gewerkschaft vergleichsweise ungünstig ausfallen, als Bedrohung angestammter Majoritätsverhältnisse erlebt werden und dadurch zur Konflikt-Eskalation beitragen.

Auf der methodischen Ebene impliziert die Analyse der Konflikteskalation zwischen Gruppen insofern eine Mehrebenenanalyse, die wir im Rahmen dieser Untersuchung jedoch nicht durchführen konnten. Weitere Forschung ist daher notwendig, um die von uns angenommene Bedeutung von Beziehungskonflikten einerseits und der Induktion 
von Minoritäten-/Majoritätenproblemen andererseits im Rahmen der (Diversity vermitttelten) Konflikteskalierung zu präzisieren.

In Ergänzung zur bisherigen Unterscheidung zwischen einer sichtbaren und einer nicht sichtbaren Diversity ${ }^{90}$ sowie zwischen einer Aufgaben-affinen und einer Beziehungs- bzw. Emotions-affinen Diversity ${ }^{91}$ regen wir deshalb an, zukünftig tabuisierte von nicht-tabuisierten Diversity-Formen zu unterscheiden. Ein organisational relevanter, tendenziell tabuisierter Diversity-Inhalt liegt in den USA z. B. in immer wieder behaupteten Intelligenzunterschieden zwischen Farbigen und Weißen ${ }^{92}$, in Deutschland z. B. in Gehaltsunterschieden, schichtabhängigen Unterschieden in den Beförderungschancen und in Verhaltensunterschieden zwischen Jugendlichen mit und ohne Migrationshintergrund. Je mehr sich derartige mehr oder weniger tabuisierte Unterschiede mit Gefühlen wie Neid und Rivalitäten verbinden, die ihrerseits tabuisiert $\operatorname{sind}^{93}$, desto eher könnte durch weitere Forschung untersucht werden, auf welche nicht-tabuisierten Konfliktinhalte derartige tabuisierte Unterschiede - möglicherweise sozial gebilligt - verschoben werden. Dies setzt im ersten Schritt jedoch die Untersuchung der Frage voraus, welche Diversity-Inhalte in Organisationen tabuisiert und welche nicht-tabuisiert sind. Vorliegende Forschungen zu Diskriminierungen und Stigmatisierungen ${ }^{94}$ können hierzu Hinweise geben.

Dabei ergibt sich allerdings das Problem, wie methodisch eine valide Zuordnung eines Diversity-Inhalts zu den Kategorien „tabuisiert" bzw. „nicht-tabuisiert“ gewährleistet werden kann. Es ist daran zu erinnern, dass die Tabuisierung eine Frage des Grades darstellt, Übergänge also fließend sind. Zu berücksichtigen ist außerdem, dass gerade in den Bereichen, in denen Tabus existieren, der Tatbestand eines Tabus als solcher ebenfalls tabuisiert sein dürfte. Bestimmte Beobachtungen valide im Sinne von Tabuindikatoren interpretieren zu können, wird deswegen die Hilfe außenstehender Experten unabdingbar machen.

\subsection{Implikationen für das Management}

Wir hatten einleitend herausgestellt, dass es uns nicht primär un die Besonderheiten der in südindischen Schulen tabuisierten bzw. nicht-tabuisierten Konfliktfelder geht, sondern in heuristischer Absicht um die Erhellung der im Prinzip generell - in Kerala und außerhalb Keralas - wirksamen Dynamik, mit der sich tabuisierte und nicht-tabuisierte Inhalte in unterschiedlicher Weise in Konflikte übersetzen. Wir sind also mit der Frage konfrontiert, ob bzw. in welchem Sinn die hier vorgetragenen Überlegungen und Befunde auch außerhalb des in Kerala untersuchten Kontextes gelten könnten. Wir haben in Kerala Bedingungen der Entstehung von Konfliktverschiebungen und Konflikteskalationen im Zuge demografischer Diversity präzisiert. Wir werden nachstehend zu klären versuchen, inwieweit mit diesen Bedingungen - wenngleich bezogen auf andere Arten der Diversity - auch in Deutschland zu rechnen ist. Erst im zweiten Schritt fragen wir dann, was das Vorliegen dieser Bedingungen für das Diversity Management in Deutschland bedeutet. Es versteht sich von selbst, dass die nachstehenden Ausführungen unter einem erheblichen Vorbehalt stehen. Wir beginnen mit dem Problem der Konflikteskalation. 
a. Bedingungen und Management von Konflikteskalation

Wir hatten als Bedingungen einer Konflikteskalierung auf die beiden voneinander unabhängigen Faktoren der Induktion von Beziehungskonflikten und der Auslösung von Minoritäten-/Majoritätenproblemen verwiesen und gezeigt, wie sich diese Faktoren in eine Konflikteskalation zwischen Gruppen übersetzen können. Wir behandeln diese beiden in Kerala parallel wirksamen Faktoren hier getrennt, um die Gedankenführung zu erleichtern.

Induktion von Beziehungskonflikten

Treffen in einer Projektgruppe die Repräsentanten unterschiedlicher Funktionen aufeinander (funktionale Diversity), so ergibt sich ein Dissens in Bezug auf die Frage, wie das jeweilige Problem am besten zu lösen ist. Dieser Dissens ist betrieblicherseits auch ausdrücklich gewollt - also nicht tabuisiert -, um so z. B. Synergien und die Abgestimmtheit von Maßnahmen zu fördern. Da die funktionale Diversity oft mit einer unterschiedlichen „Denkwelt" 95 der Funktionsrepräsentanten (z. B. Marketing und Produktion) einhergeht, kann diese Debatte durchaus hitzig verlaufen. Wir denken, dass diese Konstellation mit der Diskussion von Lehrern in Kerala, die verschiedenen Gewerkschaften angehören und über die beste Unterrichtsgestaltung diskutieren, tendenziell vergleichbar ist. Die Zugehörigkeit zu Funktions- bzw. Gewerkschaftsgruppen und damit zu spezifischen Denkwelten ${ }^{96}$ bzw. Gewerkschaftsorientierungen begünstigt, dass die Dissenspräzisierung in beiden Fällen in Beziehungskonflikte übergeht ${ }^{97}$, die Spannungen zwischen den Subgruppen intensivieren können.

Damit stellt sich die Frage, wie der positive Zusammenhang zwischen Aufgabendissens und Beziehungskonflikten abgeschwächt werden kann ${ }^{98}$ Aus den hierzu vorliegenden Befunden ergibt sich als Konsequenz für das Management dieser nicht tabuisierten (hier: funktionalen) Diversity, dass der Manager in dieser Projektgruppe eine sogenannte gemeinsame soziale Identität ${ }^{99}$ aufzubauen versuchen sollte, so dass sich die Funktionsinhaber weniger mit der Herkunftsfunktion und mehr mit der Projektgruppe identifizieren. Hierzu ist es hilfreich, die Projektgruppenmitglieder nicht abhängig von ihren individuellen Teilleistungen, sondern von dem Projekgruppenerfolg als Ganzem zu belohnen. ${ }^{100}$ Die Herausarbeitung eines gemeinsamen Zieles, unterstrichen durch eine die Gemeinsamkeit betonende transformationale Führung ${ }^{101}$, fördert die Entwicklung von wechselseitigem Vertrauen, das den Zusammenhang zwischen Aufgabendissens und Beziehungskonflikten nachweislich abschwächt. ${ }^{102}$ (Wir denken, dass diese Strategie im Prinzip auch zur DeEskalation in den untersuchten Lehrerkollegien Keralas beitragen könnte).

\section{Induktion von Minoritäten-/Majoritätenproblemen}

Minoritäten-/Majoritätenprobleme stellten sich in Kerala, wenn die quantitative (Um)Verteilung im Zuge zunehmender Diversity zu Lasten einer Gruppe geht, die bisher bzw. ansonsten innerhalb oder außerhalb der Einheit einen Majoritätsstatus innehatte. Dies gilt in Deutschland z. B. in Bezug auf die politisch gewollte zunehmende GeschlechtsDiversity, die zu Lasten der männlichen Organisationsmitglieder geht und von diesen entsprechend mit Vorbehalten aufgenommen wird. Managementbezogen zeigen empirische Analysen von ,affirmative action programs“ in den USA ${ }^{103}$, dass Quotenvorgaben zugunsten von Frauen im Unterschied etwa zu Qualifizierungsprogrammen zugunsten 
von Frauen innerbetrieblich den relativ größten Widerstand auslösen. Quotenvorgaben werden in den USA als Verletzung sowohl der Leistungs- als auch der Fairness-Norm erlebt. ${ }^{104}$ Erlässt der Betrieb Regeln zur Förderung der Geschlechts-Diversity, so kommt es für die Förderung der Regelakzeptanz auf die Befristung derartiger Maßnahmen, auf eine unzweideutige Kommunikation des Inhalts der Regelwerke und auf die Förderung sog. prozeduraler Fairness an. ${ }^{105}$ Dies wiederum impliziert, dass die konfligierenden Parteien in die Festlegung der Regelwerke integriert werden (Partizipation bei der Festlegung) und man später auch tatsächlich gemäß dieser gemeinsamen Festlegungen verfährt (Transparenz der Einhaltung gemeinsamer Regelwerke). ${ }^{106}$ (Diese Maßnahmen würden in Kerala eher nicht greifen, da dort politisch nicht eine zunehmende gewerkschaftiche Diversity, sondern die Vormachtstellung der KSTA gewollt ist).

b. Bedingungen und Management von Konfliktverschiebung

Nicht nur in kulturell geschlossenen Gesellschaften, sondern auch in offenen Gesellschaften ist mit dem Vorhandensein von Tabus zu rechnen. ${ }^{107}$ Diversity-bezogen vermuten wir, dass die migrationsbedingte Zunahme sowohl der Nationen/Ethnien-Diversity als auch der kulturellen/religiösen Diversity in Deutschland zumindest eine Tabu-Affinität aufweist. Es fällt z. B. auf, dass bereits die Verwendung des Begriffs ,Rasse" in Deutschland -im Unterschied zu den USA- mit einem Tabu belegt ist und in der offiziellen Sprache durch den Begriff Ethnie ersetzt wurde. Sprachanalysen dürften ein interessantes Vehikel der Tabu-Diagnosen darstellen. ${ }^{108}$ Sie werden bisher nur wenig eingesetzt. Auch in unserem Kontext sind Methoden der Tabu-Diagnostik nicht entwickelt, so dass nachstehende Überlegungen auf Plausibilitätsannahmen beruhen.

Die in den Massenmedien in Deutschland diskutierte These einer zunehmenden migrationsbedingten Veränderung der bundesrepublikanischen Kultur speziell in der Langzeitperspektive, die Diskussion um die Parallelgesellschaften usw. ist den Organisationsangehörigen genauso bekannt, wie den Lehrern in Kerala die politische Diskussion um die Ziele religiöser Minderheiten bekannt ist. Die innerbetriebliche Diskussion speziell der möglichen Negativeffekte einer migrationsbedingten Zunahme der Nationen/ Ethnie-Diversity und der kulturellen/religiösen Diversity mit den je betroffenen Gruppierungen würde deswegen wohl als taktlos und unsensibel zu qualifizieren sein. Da das Risiko einer Konfliktinduktion entsprechend erheblich wäre, werden derartige Debatten gerade in For profit-Organisationen, deren Erfolg von gedeihlicher Zusammenarbeit lebt, - vermutlich unausgesprochen - mit einem Tabu belegt. Aufgrund der besonderen Identitäts-Relevanz der religiösen Dimension ist speziell die Zunahme der religiösen Diversity ein Problemfeld, dessen innerbetriebliche Diskussion - siehe den Streit um das Tragen des muslimischen Kopftuchs - Risiken birgt. ${ }^{109}$ Über Tabuisierung und Segregation werden offene Konflikte zwischen den jeweiligen Subgruppen unterlaufen. Dies bedeutet aber mitnichten, dass innerbetrieblich zwischen den jeweiligen Gruppierungen keine Vorbehalte bestünden. Da über Tabuisierungen und Segregation (s. o.) Korrekturen von Vorurteilen systematisch behindert werden, können latente negative Emotionalitäten sogar u. U. noch vergrößert werden. Ihre Tabu-bedingte Unartikulierbarkeit begünstigt Konfliktverschiebungen auf nicht-tabuisierte Konfliktfelder.

Wir denken deswegen, dass - wie in Kerala - auch in Deutschland Tabuisierungen und damit Konfliktverschiebungen - hier: im Zuge der Zunahme der genannten migra- 
tionsbedingten Formen von Diversity - zu erwarten sind. Dies bedeutet zum einen, dass Konflikte dort entstehen, wo sie sonst nicht entstehen würden bzw. Konflikte an unverhältnismäßiger Schärfe gewinnen und zum zweiten, dass Manager bei der Suche nach den Konfliktursachen in die Irre geleitet werden können und gerade dadurch noch zur Konfliktverschärfung beitragen.

Zur Abmilderung der Probleme im Zuge wachsender Nationen-/Ethnien- und kultureller/religiöser Diversity wird dem Management häufig das Konzept des Multikulturalismus empfohlen. ${ }^{110}$ Dieses Konzept, in dem Toleranz und Diversity als Werte an sich betrachtet werden, kann im günstigen Fall negative Emotionalitäten zwischen Subgruppen zumindest abschwächen und so den Anlass für Konfliktverschiebungen reduzieren. "' Diese Strategie stößt innerbetrieblich jedoch auf zwei Barrieren. Multikulturalismus schließt, da Diversity als Wert an sich positiviert wird, eine Förderung und damit Zunahme jeglicher Diversity ein. Dies ist für die Betriebe aber kaum akzeptabel, wenn nicht zumindest indirekte positive Effekte einer gesteigerten Diversity auf die betriebliche Leistung zu erwarten sind. Diese Anforderung gilt vermutlich weniger in den Non profit-Schulen Keralas, ausdrücklicher aber in For profit-Unternehmen. Eben diese Bedingung positiver Leistungseffekte wird durch die vorliegende Forschung zu den Effekten einer angehobenen Nationen-/Ethnie- und kulturellen/religiösen Diversity - ähnlich wie in Bezug auf die Geschlechts-Diversity - eher nicht bestätigt; die empirischen Befunde sind zumindest sehr widersprüchlich. ${ }^{112}$ Die innerbetriebliche Forcierung der Idee des Multikulturalismus kann darüber hinaus sogar kontraproduktive Effekte freisetzen. ${ }^{113}$ Wie erwähnt, tendiert die jeweilige Mehrheit - durchaus interessengeleitet - zur Gegenstrategie der Assimilation $^{114}$, so dass die Betonung von Multikulturalismus innerhalb der Organisation negative Emotionalitäten zwischen Subgruppen bzw. Mehrheiten und Minderheiten tendenziell nicht abschwächt, sondern intensiviert.

Statt die Problemlage einer Tabuisierung und Konfliktverschiebung in ForProfit-Organisationen durch Multikulturalismus-Programme noch $\mathrm{zu}$ verschärfen, scheint es uns aussichtsreicher zu sein, den Spreng- und zentrifugalen Kräften der (zumindest partiell) tabuisierten Diversity zentripetale Kräfte entgegenzustellen. Dabei räumen wir dem Versuch Chancen ein, auf den oben beschriebenen Wegen eine gemeinsame soziale Identität vor allem auf Unternehmensebene zu entwickeln. ${ }^{115}$

\section{Anmerkungen}

1 Vgl. Van Knippenberg und Schippers (2007, S. 515).

2 Vgl. Mor Barak (2010).

3 Vgl. Jackson und Joshi (2010).

4 Vgl. Gebert (2004); Van Knippenberg und Schippers (2007, S. 518 ff.).

5 Vgl. Van Knippenberg et al. (2004, S. 1009).

6 Vgl. Tajfel und Turner (1986); Jackson et al. (2003, S. 806 ff.).

7 Vgl. Harrison et al. (2002, S. 130 ff.).

8 Vgl. Jackson et al. (2003, S. 814 ff.).

9 Vgl. Krajweski und Schröder (2008). 
10 Vgl. Krajweski und Schröder (2008).

11 Vgl. Jackson et al. (2003, S. 804).

12 Vgl. Van Knippenberg und Schippers (2007).

13 Vgl. Chhokar (2003).

14 Vgl. Harrison und Klein (2007, S. 1200).

15 Vgl. Harrison und Klein (2007, S. 1203).

$16 \mathrm{Vgl}$. Konrad und Linnehan (1995).

17 Vgl. Kumar und George (2009).

18 Vgl. Kumar und George (2009).

19 Vgl. Gebert et al. (2011).

20 Vgl. Gläser und Laudel (2009).

21 Vgl. Menon (2010).

22 Vgl. Menon (2010).

23 Vgl. mündliche Auskunft des Office of the Director for Public Instruction.

24 Vgl. mündliche Auskunft des Office of the Director for Public Instruction.

25 Vgl. mündliche Auskunft des Office of the Director for Public Instruction.

26 Vgl. Kumar und George (2009).

27 Vgl. Kumar und George (2009).

28 Vgl. The Hindu (2009).

29 Vgl. The Hindu (2006).

30 Vgl. Devika (2008).

31 Vgl. Jansamachar.net (2009).

32 Vgl. Jansamachar.net (2009).

33 Vgl. Census (2011).

34 Vgl. Menon (2010).

$35 \mathrm{Vgl}$. Menon (2010).

36 Vgl. Das (2003); Kumar und George (2009).

37 Vgl. Burrell (1984).

38 Vgl. Vidaillet (2007).

39 Vgl. Sievers und Merski (2006).

40 Vgl. Krajewski und Schröder (2008).

41 Vgl. Kakar und Kakar (2007); Michael (2009).

42 Vgl. Chhokar (2003).

43 Vgl. Shah et al. (2006); Gorringe (2005).

44 Vgl. Chhokar (2003).

45 Vgl. Kumar und George (2009).

46 Vgl. Kumar und George (2009).

47 Vgl. Chhokar (2003).

48 Vgl. Chhokar (2003).

49 Vgl. Ruback und Singh (2008).

50 Vgl. Lobo (2002, S. 115 ff.). 
51 Vgl. Menon (2010).

52 Vgl. Kumar und George (2009).

53 Vgl. Census (2011).

54 Vgl. India.gov.in (2009).

55 Vgl. Menon (2010).

56 Vgl. Das (2003, S. 3234 f.).

57 Vgl. Devika (2008); Wellman und Tokuno (2004).

58 Vgl. Jehn (1995).

59 Vgl. Oakes et al. (1994).

$60 \mathrm{Vgl}$. Brewer und Brown (1998).

61 Vgl. Simons und Peterson (2000, S. 108).

62 Vgl. Jehn (1995).

63 Vgl. Mooney et al. (2007, S. 733 ff.).

64 Vgl. Pruitt und Kim (2004).

65 Vgl. Verkuyten (2005), 129; Wolsko et al. (2006, S. 301).

66 Vgl. Tajfel (1981).

67 Vgl. Wellman und Tokuno (2004, S. 292 ff.).

68 Vgl. Beyer (2003, S. 336 f.).

69 Vgl. Tajfel (198I).

70 Vgl. Wuthnow.(2007)

71 Vgl. Wuthnow (2007)

72 Vgl. Devika (2008).

73 Vgl. Wuthnow (2007).

74 Vgl. Blau (1977).

75 Vgl. Jehn (1995)

76 Vgl. Jehn und Mannix (2001).

77 Vgl. Brislin (1980).

78 Vgl. James et al. (1984, S. 874 ff.).

79 Vgl. Bliese (2000)

80 Vgl. Bantel und Jackson (1989).

81 Vgl. Milliken und Martins (1996).

82 Vgl. Harrison und Klein (2007).

83 Für die Modelle 2,4,5 und 6 schwanken die Variance Inflation Factors zwischen 1.087 und 1.231 .

84 Vgl. Van Knippenberg und Schippers (2007, S. 515 ff.).

85 Vgl. Harrison et al. (2002, S. 1029 ff.).

86 Vgl. Gersick (1991).

87 Vgl. Behar et al. (2008)

88 Vgl. De Dreu und Weingart (2003); Mooney et al. (2007, S. 736 ff.).

89 Vgl. Behar et al. (2008)

90 Vgl. Harrison et al. (2002, S. 1030 ff.). 
91 Vgl. Pelled (1996, S. 615 ff.).

92 Vgl. Nisbett (2009).

93 Vgl. Vidaillet (2007).

94 Vgl. Konrad und Linnehan (1995); Krell et al. (2007).

95 Vgl. Dougherty (2001, S. 613).

96 Vgl. Dougherty (2001, S. 613).

97 Vgl. De Dreu und Weingart (2003).

98 Vgl. Gebert (2004, S. 418 f.).

99 Vgl. Kane et al. (2005).

$100 \mathrm{Vgl}$. Schippers et al. (2003, S. 799).

101 Vgl. Kearney und Gebert (2009); Hüttermann und Boerner (in Druck).

102 Vgl. Simons und Peterson (2000, S. 107).

$103 \mathrm{Vgl}$. Harrison et al. (2006).

$104 \mathrm{Vgl}$. Harrison et al. (2006, S. 1014).

105 Vgl. Harrison et al. (2006, S. 1030).

106 Vgl. Cropanzano und Stein (2009).

107 Vgl. Krajweski und Schröder (2008)

$108 \mathrm{Vgl}$. Krajweski und Schröder (2008).

109 Vgl. Wuthnow (2007); Gebert und Kearney (2010).

110 Vgl. Stevens et al. (2008, S. 120 ff.).

111 Vgl. Wuthnow (2007).

112 Vgl. Jackson et al. (2003, S. 809 ff.).

$113 \mathrm{Vgl}$. Stevens et al. (2008, S. 121 f.).

114 Vgl. Verkuyten (2005); Wolsko et al. (2006)

115 Vgl. Hogg und Terry (2000, S. 13I ff.).

\section{Literatur}

Bantel K, Jackson SE (1989) Top management and innovations in banking: does the composition of the top team make a difference? Strategic Manage J 10(Suppl 1):107-124

Behar KJ, Peterson RS, Mannix EA, Trochim WMK (2008) The critical role of conflict resolution in teams: a close look at the links between conflict type, conflict management strategies, and team outcomes. J Appl Psychol 93:170-188

Beyer P (2003) Constitutional privilege and constituting pluralism: religious freedom in national, global, and legal context. J Sci Study Religion 42:333-339

Blau PM (1977) Inequality and heterogeneity: a primitive theory of social structure. The Free Press, New York

Bliese P (2000) Within-group agreement, non-independence, and reliability: implications for data aggregation and analysis. In: Klein KK, Kozlowski SW (Hrsg) Multilevel theory, research, and methods in organizations. Jossey-Bass, San Francisco, S 349-381

Brewer MB, Brown RJ (1998) Intergroup relations. In: Gilbert DT, Fiske ST, Lindzey G (Hrsg) Handbook of social psychology. McGraw-Hill, Boston, S 554-594 
Brislin RW (1980) Translation and content analysis of oral and written material. In: Triandis HC, Berry JW (Hrsg) Handbook of cross-cultural psychology. Allyn \& Bacon, Boston, S 349-444

Burrell G (1984) Sex and organizational analysis. Org Stud 5(2):97-118

Census (2011) http://www.censusindia.gov.in/Census Data 2011/India at glance. Zugegriffen: 19. Mai 2011

Chhokar JS (2003) India: Diversity and complexity in action. In: Chhokar JS, Brodbeck FC, House RJ (Hrsg) Culture and leadership across the world: the GLOBE book of in-depth studies of 25 societies. Psychological Press, New York, S 971-1020

Cropanzano R, Stein JH (2009) Organizational justice and behavioral ethics: promises and prospects. Bus Ethics Q 19(2):193-233

Das MK (2003, Aug 2) Politics of pressure groups. Economic \& Political Weekly, 3234-3235

De Dreu CK, Weingart LR (2003) Task versus relationship conflict, team performance and team member satisfaction: a meta-analysis. J Appl Psychol 88:741-789

Devika J (2008, Juli 26) Memory's fatal lure: the left, the congress and 'Jeevan' in Kerala. Economic \& Political Weekly, 13-16

Dougherty D (2001) Reimagining the differentiation and integration of work for sustained product innovation. Org Sci 12:612-631

Gebert D (2004) Durch Diversity zu mehr Teaminnovativität? Ein vorläufiges Resümee der empirischen Forschung sowie Konsequenzen für das Diversity Management. Betriebswirtschaft $4: 412-430$

Gebert D, Boerner S, Chatterjee D (2011) Do religious differences matter? An analysis in Indian organizations. Team Performance Management 17(3/4):224-240

Gebert D, Kearney E (2010) Religious Diversity in organizations: a relevant, but neglected dimension. Paper presented at the Academy of Management Meeting, Montreal, August 9th-- 10th

Gersick CJG (1991) Revolutionary change theories: a multilevel exploration of the punctuated equilibrium paradigm. Acad Manag Rev 16:10-36

Gläser J, Laudel G (2009) Experteninterviews und qualitative Inhaltsanalyse, 3. Aufl. VS Verlag für Sozialwissenschaften, Wiesbaden

Gorringe H (2005) Untouchable citizens: Dalit movements and democratization in Tamil Nadu. Sage, New Delhi

Harrison DA, Klein KJ (2007) What's the difference? Diversity constructs as separation, variety, or disparity in organizations. Acad Manag Rev 32(4):1199-1228

Harrison DA, Price KH, Gavin JH, Florey AT (2002) Time, teams, and task performance: changing effects of surface- and deep-level Diversity on group functioning. Acad Manag J 45:1029-1045

Harrison DA, Mayer DM, Kravitz DA (2006) Understanding attitudes toward affirmative action programs in employment: summary and meta-analysis of 35 years of research. J Appl Psychol 91(5):1013-1036

Hogg MA, Terry DJ (2000) Social identity and self-categorization processes in organizational contexts. Acad Manag Rev 25:121-140

Hüttermann H, Boerner S (in press) Fostering innovation in functionally diverse teams: the two faces of transformational leadership. European J Work Organizational Psychol

India.gov.in (2009, Aug 3) Know India, National Portal

Jackson SE, Joshi A (2010) Work team Diversity. In: Zedeck S (Hrsg) APA handbook of industrial and organizational psychology, Bd I. American Psychological Association, Washington, S $651-686$

Jackson SE, Joshi A, Erhardt NL (2003) Recent research on team and organizational Diversity: SWOT analysis and implications. J Manag 29:801-830

James LR, Demaree RG, Wolf G (1984) Estimating within-group interrater reliability with and without response bias. J Appl Psychol 69:85-98

Jansamachar.net (2009, Aug 3) Online newspaper in English \& Hindi 
Jehn KA (1995) A multimethod examination of the benefit and detriments of intragroup conflict. Adm Sci Q 40:256-282

Jehn KA, Mannix EA (2001) The dynamic nature of conflict: A longitudinal study of intragroup conflict and group performance. Acad Manage J 44(2):238-251

Kakar S, Kakar N (2007) The Indians: portrait of people. Penguin, New York

Kane A, Argote L, Levine JM (2005) Knowledge transfer between group via personnel rotation: effects of social identity and knowledge quality. Organ Behav Hum Decis Process 96:56-71

Kearney E, Gebert D (2009) Managing Diversity and enhancing team outcomes: the promise of transformational leadership. J Appl Psychol 94(1):77-89

Konrad AM, Linnehan F (1995) Race and sex differences in line with managers reactions to equal employment opportunity and affirmative action interventions. Group Org Stud 20:409-439

Krajweski S, Schröder H (2008) Silence and taboo. In: Antos G, Ventola E (Hrsg) Handbook of interpersonal communication. Mouton de Gruyter, New York, S 595-619

Krell G, Riedmüller B, Sieben B, Vinz D (2007) Diversity studies. Grundlagen und disziplinäre Ansätze. Campus, Frankfurt

Kumar NA, George KK (2009, Okt 10) Kerala's education systems: from inclusion to exclusion? Economic \& Political Weekly, 55-61

Lobo L (2002) Persecution of Indian Christians. Dialog: A J Theol 41(2):114-122

Menon NR (2010, Aug 21) Imagined Kerala. Economic \& Political Weekly, 22-25

Michael A (2009) Der Hinduismus: Geschichte und Gegenwart. Beck, Stuttgart

Milliken FJ, Martins LL (1996) Searching for common threads: understanding the multiple effects of Diversity in organizational groups. Acad Manag Rev 21:402-433

Mooney ACP, Holahan PJ, Amason AC (2007) Don't take it personally: exploring cognitive conflicts as a mediator of affective conflict. J Manag Stud 44:733-758

Mor Barak ME (2010) Managing Diversity: toward a globally inclusive workplace, 2. Aufl. Sage Publications, Thousand Oaks

Nisbett RE (2009) Intelligence and how to get it: why schools and cultures count. W. W. Norton \& Co, New York

Oakes PJ, Haslam SA, Turner JC (1994) Stereotyping and social reality. Blackwel, Malden

Pelled LH (1996) Demografic Diversity, conflict, and work group outcomes: an intervening process theory. Org Sci 7:615-631

Pruitt DG, Kim SH (2004) Social conflict: escalation, stalemate, and settlement. McGraw-Hill, New York

Ruback RB, Singh P (2008) Inequity in Hindu-Muslim-riots: a test of two biases. J Appl Psychol 4:982-998

Schippers MC, Den Hartog DN, Koopman PL, Wienk JA (2003) Diversity and team outcomes: the moderating effect of outcome interdependence and group longevity and the mediating effect of reflexivity. J Organ Behav 24:779-802

Sievers B, Mersky RR (2006) The economy of vengeance: some considerations on the aetiology and meaning of the business of revenge. Hum Relat 59(2):241-259

Shah G, Mander H, Thorat S, Deshpande S, Baviskar A (2006) Untouchability in rural India. Sage, New Delhi

Simons TL, Peterson RS (2000) Task conflict and relational conflict in top management teams: the pivotal role of intragroup trust. J Appl Psychol 85(1):102-111

Stevens FG, Plaut VC, Sanchez-Burks J (2008) Unlocking the benefits of Diversity: allinclusive multiculturalism and positive organizational change. J Appl Behav Sci 1:116-133

Tajfel H (1981) Human Groups and Social Categories: studies in social psychology. Cambridge University Press, Cambridge

Tajfel H, Turner JC (1986) Social identity theory and intergroup behavior. In: Worchel S, Austin WG (Hrsg) Psychology of intergroup relations. Nelson-Hall, Chicago, S 7-24

The Hindu, 10.2.(2006) 
The Hindu, 1.2.(2009)

Van Knippenberg D, De Dreu CKW, Homan AC (2004) Work Group Diversity and Group Performance: an Integrative Model and Research Agenda. J Appl Psychol 89(6):1008-1022.

Van Knippenberg D, Schippers MC (2007) Work group Diversity. Annu Rev Psychol 58:515-541

Verkuyten M (2005) Ethnic group identification and group evaluation among minority and majority groups: testing the multiculturalism hypothesis. J Personell Soc Psychol 88:121-138

Vidaillet B (2007) Lacanian theory's contribution to the study of workplace. Hum Relat 11:1669-1700

Wellman JK, Tokuno K (2004) Is religious violence inevitable? J Sci Stud Religion 43:291-296

Wolsko C, Park B. Judd CM (2006) Considering the tower of Babel: correlates of Assimilation and multiculturalism among ethnic minority and majority groups in the United States. J Soc Justice Res 19(3):277-306

Wuthnow R (2007) America and the Challenges of religious Diversity. Princeton University Press, Princeton

\section{Should Diversity be openly discussed or not? An explorative study in India}

Abstract: Completing existing forms of diversity, the paper is the first to introduce the distinction between an off-limits form of diversity and a non off-limits form of diversity. This distinction is relevant because we argue that an off-limits form of diversity will result in a shift of intragroup conflicts, while a non off-limits form of diversity will result in an escalation of intragroup conflicts. For the management of diversity, this distinction is important to appropriately assess the risks of rising diversity in organizations. Analyzing data from $\mathrm{N}=96$ schools in southern India (Kerala), religious diversity appears to be off-limits, whereas union diversity does not. As expected, union diversity was accelerated positively related to intragroup conflicts. Moreover, this positive relationship was stronger under high levels of religious diversity. In order to explain these results, we explicitly consider the particularites of Kerala. In order to draw conclusions for diversity management in German organizations, we additionally analyze which forms of diversity could unfold analogous dynamics and what implications for managers are to expect.

Keywords: Religious diversity · Union diversity · Taboo · Diversity management 